\title{
Causal or Casual?-The Association Between Consumption of Artificially Sweetened Carbonated Beverages and Vascular Disease
}

\author{
Shuchi Anand, MD and Wolfgang C. Winkelmayer, MD, SCD \\ Division of Nephrology, Department of Medicine, Stanford University School of Medicine, Palo Alto, CA, USA.
}

J Gen Intern Med 27(9):1100-1

DOI: $10.1007 / \mathrm{s} 11606-012-2126-1$

(C) Society of General Internal Medicine 2012

$\mathrm{T}$ he United States leads the world in per capita consumption of carbonated soft drinks. On average, each American consumed 714 eight-ounce servings of "soda pop" in 2011. ' Given their widespread use, it is in the public's interest to understand any potential health outcomes from the consumption of these beverages, and as a result, an increasing number of studies have examined the association between the use of carbonated soft drinks and a variety of health outcomes.

In this issue of the Journal of General Internal Medicine, Gardener et al. report on the association between soft drink consumption and vascular events in the diverse, populationbased cohort of the Northern Manhattan Study (NOMAS). ${ }^{2}$ Their surprising finding that regular consumption of artificially-sweetened (diet) soft drinks, but not sugarsweetened soft drinks, was associated with an increased risk of vascular events deserves further exploration.

A causal association between increased consumption of sugar-sweetened soda beverages is biologically plausible and supported by the literature. Several prospective cohort analyses have demonstrated an increased risk for coronary heart disease $\mathrm{e}^{3,4}$ and diabetes ${ }^{5,6}$ among persons who regularly consume sugar-sweetened soft drinks. For example, in the Nurses' Health Study, the relative risk for fatal or nonfatal myocardial infarction increased by $35 \%$ per two serving increase in sweetened soft drinks consumption. ${ }^{3}$ Remarkably, women who began to consume one or more soft drinks a day gained four kilograms over a four-year period and experienced an $80 \%$ increase in risk for type 2 diabetes. ${ }^{6}$ Similar results were observed among men in the Health Professionals Follow Up study.,

Given the observed weight gain in the Nurses' Health Study and other studies demonstrating a higher total energy intake (i.e., indicating that the drinks are not being used as calorie substitutes, but rather are calorie-additive) among persons regularly consuming sugar-sweetened soft drinks, ${ }^{7}$

Published online June 13, 2012 we can easily grasp the biological plausibility of this association. Another proposed causal mechanism points to the high glycemic load of the soft drink which predisposes to insulin resistance. ${ }^{8}$

In light of this evidence, it is curious that Gardener's et al. analysis did not demonstrate a significant increase in vascular events among persons drinking daily sugarsweetened beverage in their overall cohort. Statistical power may have been an issue. The NOMAS study followed 2,564 for 10 years, with 591 vascular events; in comparison, the Nurses Health Study involved 88,520 women followed for 24 years with 3,105 incident coronary events. An alternative explanation for the null finding may be due to the fact that soda consumption was only ascertained at baseline, with an uncertain but possibly large amount of person-time being misclassified with regard to the exposure of interest. However, consistent with other studies the authors do report a $57 \%$ increase in risk for vascular events among nonobese persons without metabolic syndrome or diabetes. The novel finding in this subgroup is the association with a composite outcome that included stroke in addition to myocardial infarction, indicating an even broader increase in risk of chronic diseases with consumption of sugar-sweetened soft drinks than has been previously acknowledged.

But what about diet soft drinks? Can regular consumption of this no-calorie substitute with presumably no direct pathway to weight gain lead to adverse health outcomes? Some nutrition experts have suggested so, particularly due to correlation in time of the obesity epidemic with rising consumption of diet soft drinks. ${ }^{9,10}$ Putative mechanisms for this include activation of intestinal "sweet taste" receptors by the artificial sweeteners which in turn purportedly increase glucose uptake. Davidson et al. proposed that the artificial sweeteners inhibit the association between sweet taste and caloric intake, such that rats fed artificial sweeteners are unable to put the brakes on intake of sweet food because they do not associate the sweet food with calories. $^{11}$

Analysis from the Multi-Ethnic Study of Atheroscelorosis cohort demonstrated an increase in risk for incident metabolic syndrome and type 2 diabetes with consumption of at least one diet soft drink daily. ${ }^{12}$ Upon adjustment for 
baseline body mass index the associations attenuated, although remained significant for the increase in incident type 2 diabetes. In the larger Health Professionals Follow Up study, the crude association between regular consumption of diet soft drink and type 2 diabetes disappeared completely on multivariate analyses adjusting for baseline body mass index. ${ }^{5}$ Similarly null results have been observed in analyses for incident coronary heart disease in the Health Professionals Follow Up study and the Nurses' Health Study. ${ }^{3,4}$

Thus although there is epidemiological and some biologic suggestion for a link between diet soft drink consumption and adverse health outcomes, the preponderance of evidence from large cohort analyses has not been supportive. Attenuation of the crude association on adjustment for body size in several of these analyses suggests that diet soft drink intake may be a marker for persons at higher risk for adverse health outcomes, rather than a causal factor.

The current NOMAS analysis, though suggestive of a modest association, does not provide convincing evidence for causation for several reasons. First, compared with persons consuming less than one serving a month, persons consuming diet soft drinks daily demonstrated evidence for a tendency toward an "unhealthy" lifestyle. They consumed more total calories and sodium; their size as captured by body mass index and waist circumference was generally larger. Second, they carried a higher burden of co-morbidities such as hypertension, peripheral vascular disease, and previous cardiac disease. Strikingly the prevalence of metabolic syndrome and diabetes was nearly $1.5-2$ fold higher in this group. Although the authors adjusted for several of these confounders, the relatively modest observed association between diet soft drink consumption and vascular events could reflect residual confounding. It would take just a weak and common or a relatively rare, yet moderately strong unobserved confounder to render the reported association null. Finally, intake of soft drinks was evaluated at baseline, with no further ascertainment of this "exposure" during the 10-year follow up period. Both the Nurses' Health and Health Professionals Follow Up studythough significantly less diverse than the NOMAS cohortlikely captured "exposure" better because of repeated measurements of beverage intake at regular intervals.

Whether regular diet soft drink consumption leads to weight gain and vascular events, via one of the putative mechanisms based of appetite stimulation, has not yet been definitely answered. It is hard to imagine a scenario where researchers could conduct a randomized, blinded, placebocontrolled trial of diet soft drink consumption. Thus the best scientific evidence for causation is likely to come from observational studies - ones that enroll participants who have similar dietary habits and co-morbidities, and rigorously capture exposure and outcomes over follow up time. The current NOMAS analysis does not satisfy all of these criteria.

In summary, the article by Gardener et al. differs from the existing evidence on this topic in two ways. While the absence of an association between sugared soda consumption and vascular events may be a result of relatively limited statistical power or exposure misclassification due to lack of updated information, we conclude that the surprising association between diet soft drink consumption and vascular events may well be spurious and certainly not conclusive or indicative of cause and effect.

Acknowledgements: Dr. Anand is supported by F32 DK 084697.

Corresponding Author: Shuchi Anand, MD; Division of Nephrology, Department of Medicine, Stanford University School of Medicine, 780 Welch Road, Suite 106, Palo Alto, CA 94304, USA (e-mail: Sanand2@stanford.edu).

\section{REFERENCES}

1. Geller M. U.S. soda consumption fell faster in 2011. 2012 Reuters. Accessed on May 17, 2012 from: http://www.reuters.com/article/2012/ 03/20/drinks-idUSL1E8EK1P620120320.

2. Gardener H, et al. Diet Soft Drink Consumption is Associated with an Increased Risk of Vascular Events in the Northern Manhattan Study. J Gen Intern Med. 2012. doi:10.1007/s11606-011-1968-2.

3. Fung TT, et al. Sweetened beverage consumption and risk of coronary heart disease in women. Am J Clin Nutr. 2009;89(4):1037-42.

4. de Koning $\mathbf{L}$, et al. Sweetened beverage consumption, incident coronary heart disease, and biomarkers of risk in men. Circulation. 2012;125 (14): 1735-41.

5. de Koning $\mathbf{L}$, et al. Sugar-sweetened and artificially sweetened beverage consumption and risk of type 2 diabetes in men. Am J Clin Nutr. 2011;93(6): 1321-7.

6. Schulze MB, et al. Sugar-sweetened beverages, weight gain, and incidence of type 2 diabetes in young and middle-aged women. JAMA. 2004;292(8):927-34

7. Vartanian LR, Schwartz MB, Brownell KD. Effects of soft drink consumption on nutrition and health: a systematic review and metaanalysis. Am J Public Health. 2007;97(4):667-75.

8. Malik VS, et al. Sugar-sweetened beverages, obesity, type 2 diabetes mellitus, and cardiovascular disease risk. Circulation. 2010;121 (11):1356-64.

9. Pepino MY, Bourne C. Non-nutritive sweeteners, energy balance, and glucose homeostasis. Curr Opin Clin Nutr Metab Care. 2011;14(4):3915.

10. Fowler SP, et al. Fueling the obesity epidemic? Artificially sweetened beverage use and long-term weight gain. Obesity (Silver Spring). 2008; 16 (8): 1894-900.

11. Davidson TL, et al. Intake of high-intensity sweeteners alters the ability of sweet taste to signal caloric consequences: implications for the learned control of energy and body weight regulation. Q J Exp Psychol (Hove). 2011;64(7): 1430-41.

12. Nettleton JA, et al. Diet soda intake and risk of incident metabolic syndrome and type 2 diabetes in the Multi-Ethnic Study of Atherosclerosis (MESA). Diabetes Care. 2009;32(4):688-94. 